\title{
Pour une approche interdisciplinaire du risque environnemental. Le cas de l'uranium
}

\author{
Sophie Bretesché ${ }^{1, *}$, Gilles Montavon ${ }^{2}$ et Alexis Martin ${ }^{3}$ \\ 1 Sociologie, IMT Atlantique, EA 4272 LEMNA, Nantes, France \\ 2 Radiochimie, IMT Atlantique, UMR 6457 Subatech, Nantes, France \\ ${ }^{3}$ Chimie, IMT Atlantique, UMR 6457 Subatech, Nantes, France
}

\begin{abstract}
Résumé - La transition écologique contemporaine impose de se pencher sur les conséquences sanitaires et environnementales du passé industriel des territoires. Le projet de recherche «Traces, Transfert, Patrimoine », dont il est question ici, s'inscrit précisément dans cette réflexion, en s'intéressant au devenir des anciennes mines d'uranium françaises. La notion de radioactivité naturelle renforcée consécutive à la post-exploitation de l'uranium rend nécessaire une compréhension de la dynamique des territoires et des contaminants, afin de contribuer à une gestion des risques plus intégrée et plus anticipatrice. Le projet s'appuie sur une démarche conduite pour appréhender le risque environnemental dans sa dimension physique et sociale. Issu d'un programme de recherche conduit entre la sociologie et la radiochimie, il vise à caractériser l'empreinte de l'uranium au travers des traces, des processus de transfert et de la qualification du patrimoine.
\end{abstract}

Mots-clés : territoires / risques / environnement / uranium / mines

\begin{abstract}
For an interdisciplinary approach to environmental risk. The case of uranium. The contemporary ecological transition makes it necessary to look into the health and environmental consequences of the industrial past on the territories. The research project is precisely part of this reflection, focusing on the future of the former French uranium mines. The notion of enhanced natural radioactivity resulting from the post-exploitation of uranium makes it necessary to understand the dynamics of territories and contaminants, in order to contribute to more integrated and more anticipatory risk management. The project is based on an approach taken to understand the environmental risk in its physical and social dimension. Resulting from a research program conducted between sociology and radiochemistry, it aims to characterize the footprint of uranium through traces, processes of transfer and qualification of heritage. Such an approach requires cross-fertilization of knowledge from radiochemistry and sociology in order to characterize the footprint of uranium mining. To answer these questions, we propose innovative research on both study objects and methodological approaches. The "Traces" axis focuses on identifying and characterizing sources of pollution, the "Transfer" axis seeks to study the transfer mechanisms governing the dispersion of pollutants and the "Heritage" axis aims to study the impacts of pollutants on different targets and to understand mechanisms for social consultation.
\end{abstract}

Keywords: territories / risks / environment / uranium / mines

En France, les débats relatifs au nucléaire portent fréquemment sur le devenir de la filière, entre arrêt des centrales ou renouvellement technique des réacteurs. Ces débats portent sur des alternatives fondées sur des scénarii prospectifs. En revanche, la gestion du passé et

\footnotetext{
* Auteur correspondant :

sophie.bretesche@imt-atlantique.fr
}

notamment celle des anciennes mines d'uranium est en partie occultée, alors même qu'elle concerne la prise en charge des restes de la production sur plusieurs centaines d'années. Si l'exploitation des sites s'est déroulée dans le contexte français sur une quarantaine d'années pendant la période de l'après-guerre, la post-exploitation requiert un suivi lié aux effets potentiels du radon et à la contamination de l'eau sur du très long terme. Comment 
prendre en compte sur le long terme l'héritage d'un passé industriel pour partie oublié ? La gestion des anciennes mines d'uranium est une question mise à l'agenda depuis la circulaire du 22 juillet 2009. Celle-ci prévoit une surveillance des anciens sites par du contrôle, une amélioration de la sécurité et de l'information mais la question de la vigilance portée aux mines se pose dans un contexte de gestion environnementale durable. Comment en effet transmettre aux générations futures cette mémoire des sites tout en maintenant la surveillance ? Cette question renvoie à la prise en compte du temps long dans les modalités de suivi des mines et plus particulièrement $\mathrm{du}$ temps de la décroissance. La temporalité spécifique associée à la radioactivité interroge en effet la façon dont nos sociétés prennent en charge dans la longue durée un héritage à risques. Comme le souligne Philippe Brunet (2004), «les temporalités et changement d'échelle contribuent à l'accroissement de l'incertitude: les quelques décades d'années de l'exploitation minière deviennent négligeables face à la durée radioactive des restes. Au fond, nul ne sait quel sera le terme de cette période.» Le projet «Traces, Transfert, Patrimoine ${ }^{1}$ » (TTP) présenté dans ce texte rend compte d'une démarche conduite pour appréhender le risque environnemental dans sa dimension physique et sociale. Conduit à l'IMT Atlantique, entre son département de sciences sociales et de gestion et le groupe Radiochimie de l'UMR Subatech, le projet de recherche vise à caractériser l'empreinte de l'uranium. Le contexte de la recherche est né d'une volonté partagée de comprendre les dimensions du risque en prenant en compte l'empreinte physique et humaine de la mine dans l'espace. En effet, la gestion post-exploitation de l'uranium en France génère d'un territoire à l'autre des réponses très diverses et un rapport au risque très différencié. Les relations entre l'uranium, son exploitation et son empreinte territoriale provoquent aujourd'hui un ensemble de controverses liées à la gestion de l'héritage et des traces laissées par l'exploitation. Cette tension requiert par conséquent de développer une prise en compte des contaminants et des caractéristiques du territoire. A partir d'un site laboratoire (La Commanderie), cette contribution présente la démarche interdisciplinaire adoptée.

\section{Des controverses contrastées autour de la qualification du risque}

Depuis les années 1990, la question de l'empreinte territoriale des mines, et plus largement des déchets, est en effet au cour de l'agenda portant sur la gestion des territoires du nucléaire (Bretesché et Ponnet, 2012;

\footnotetext{
${ }^{1}$ Ce projet s'inscrit dans le cadre du programme Pollusols (Pollutions diffuses dans le continuum Terre-Mer, 2015-2019), financé par la région Pays de la Loire, ainsi que dans le cadre de la zone atelier «Territoires uranifères de l'arc hercynien».
}

Barthe, 2006). Au fil du temps, la problématique industrielle des mines d'uranium françaises s'est effacée au profit d'une question environnementale (Brunet, 2004). La trace radioactive n'interroge pas seulement la prise en charge du passé, mais soulève aussi la question de l'anticipation et des formes de régulation du risque. L'extraction d'uranium en France (1948-2001) a concerné environ 250 sites répartis sur 27 départements. Les activités minières ont pu conduire à des contaminations diffuses dans l'environnement durant la phase d'exploitation ou encore plusieurs dizaines d'années après le réaménagement de ces sites. Celles-ci peuvent «disparaître» par effet de dilution mais peuvent également se concentrer et créer des zones singulières marquées pouvant conduire à des problèmes d'exposition (externe et/ou interne).

Ce rapport singulier à l'espace interroge plus précisément la façon dont le territoire a été modelé par l'exploitation puis la post-exploitation. En effet, au fil de l'histoire, les modalités d'appropriation et de gestion de l'espace relèvent de logiques fort distinctes, ce qui invite à prendre en compte la dimension spatiotemporelle dans l'analyse du rapport entre risque et territoire (Dauphiné et Provitolo, 2013). Les territoires uranifères constituent un exemple de gestion environnementale qui articule un niveau local et national. L'héritage uranifère est issu d'une exploitation d' «État» (Hecht, 2016) qui démarre au lendemain de la Seconde Guerre mondiale et s'arrête au début des années 2000 en raison de sa trop faible rentabilité économique. Plus de 200 sites uranifères sont répertoriés allant de la simple tranchée, d'où aucun minerai n'a été extrait, aux mines les plus importantes du Limousin (Brunet, 2004), de la Vendée et du Forez. En raison des faibles teneurs du minerai, cette industrie a laissé derrière elle plus de 200 millions de tonnes de stériles plus ou moins radioactifs et environ 50 millions de tonnes de résidus de traitement du minerai qui renferment plus de $70 \%$ de la radioactivité initiale. Ces anciennes mines constituent un héritage complexe avec de forts enjeux sociaux, sanitaires et environnementaux qui, dès le début des années 1980, ont nécessité un important travail d'expertise technique mené conjointement entre l'administration et l'exploitant pour définir les conditions d'un réaménagement préservant l'homme et l'environnement.

Dès leur fermeture, les sites miniers ont été progressivement réaménagés de sorte qu'ils retournent à un supposé état de nature; certains d'entre eux sont ceinturés de grillages. Les discrets dispositifs de contrôle de la radioactivité et la reconfiguration qui tend à effacer l'activité permettent, soit d'installer de nouveaux usages sur les terrains, soit de les laisser comme tels. C'est pourquoi, aujourd'hui, les mines d'uranium balancent entre d'un côté, l'oubli et la banalisation, et de l'autre, la mise en mémoire par les traces subsistantes. Or, «si le 
problème disparaît des mémoires, il n'est pas pour autant résolu» (Bretesché et Ponnet, 2013). En effet, au tournant des années 1990, le débat public autour de la fermeture de ces mines évolue progressivement vers la remise en cause des modalités de gestion post-exploitation. Des analyses divergentes sur les conditions des réaménagements et les options retenues apparaissent montrant la nécessité de prendre en considération d'autres intérêts et enjeux que ceux définis par les acteurs historiques de l'expertise. Le rapport de force devient visible avec des retentissements tant médiatiques que judiciaires qui vont s'égrener tout au long des trente dernières années. Cependant, l'intensité du débat relayé par la presse apparaît contrastée : si certains sites miniers du Massif central occupent le devant de la scène, ceux situés dans l'Ouest de la France et d'importance équivalente n'apparaissent paradoxalement pas dans l'espace des conflits. On y retrouve pourtant les mêmes types de vestiges, les procédures de réaménagement, de surveillance et de gestion relèvent d'un cadre réglementaire et législatif commun, les autorités sont partagées et l'exploitant est identique.

La gestion des mines d'uranium implique un traitement des risques communs à l'ensemble des sites miniers (effondrements, affaissements, régimes hydrologiques...) et demande en même temps de prendre en compte la spécificité des risques radioactifs. Ces deux aspects sont réunis dans un autre outil de gestion au service de l'aménagement développé par le gouvernement. En effet, la loi « après-mine » du 30 mars 1999 crée les plans de prévention des risques miniers $\left(\mathrm{PPRM}^{2}\right)$, considérés comme des servitudes d'utilité publique dont la procédure d'élaboration est similaire à celle des plans de prévention des risques naturels. Prescrits par le préfet, ils intègrent le risque des rayonnements ionisants. Mais, dans les faits, cet outil de maitrise de l'urbanisation concerne surtout les anciennes exploitations de fer et de houille, et ignore l'uranium ${ }^{3}$. Ainsi, la controverse qui entoure la prise en charge des déchets des sites repose à la fois sur la qualification des restes et de leur teneur en uranium et sur la caractérisation des formes d'usages et de mémoire. En fonction des territoires, la gestion du risque ne prend pas les mêmes formes (Becerra et al., 2016). Compte tenu de ce contexte, la controverse qui entoure la prise en charge des déchets requiert d'associer deux approches : d'un côté, les traces objectives laissées par l'histoire et, de l'autre, l'action de l'homme dans l'usage contemporain du territoire.

\footnotetext{
${ }^{2}$ Par la loi $\mathrm{n}^{\circ} 99-245$ relative à la responsabilité en matière de dommages consécutifs à l'exploitation minière et à la prévention des risques miniers après la fin de l'exploitation. Disponible sur: https://www.legifrance.gouv.fr/affichTexte. do?cidTexte=JORFTEXT000000759770.

${ }^{3} \mathrm{http} / / / \mathrm{www}$.georisques.gouv.fr/acces-aux-donnees-gaspar.
}

\section{Une méthode interdisciplinaire: Traces, Transfert, Patrimoine}

Le projet interdisciplinaire «Traces, Transfert, Patrimoine », développé à travers une analyse conjointe du point de vue de la radiochimie et de la sociologie, part de la nécessité de comprendre et de donner du sens aux traces laissées par l'exploitation de l'uranium sur le territoire. En effet, la fin de l'exploitation des sites et le réaménagement opéré au cours des années 1990 ont eu pour effet d'effacer progressivement les traces industrielles de l'extraction d'uranium en opérant une stricte délimitation entre les zones de surveillance et le reste du territoire. Au fil du temps, ce zonage s'est avéré contesté au titre des « restes » (stériles, résidus) qui ont révélé, en fonction des territoires et des cultures locales, des « zones de bord» (Thom, 1993), c'est-à-dire des espaces sans dispositifs de gestion du risque sur le long terme. Par conséquent, appréhender le risque requiert d'étudier la dynamique de transfert des radioéléments mais également les interactions qui ont lieu entre un environnement et ses habitants.

La méthode exploratoire interdisciplinaire est déclinée sur une mine choisie dans le cadre des sites ateliers de la ZATU (zone atelier Territoires uranifères de l'arc hercynien $\left.{ }^{4}\right)$. L'axe «Traces» s'attache ainsi à identifier et à caractériser les sources de pollution, autrement dit les points d'émission et de rejets ainsi que les traces laissées par l'exploitation industrielle. L'axe «Transfert» cherche à étudier les mécanismes de transferts régissant la dispersion des polluants (du compartiment « eau» vers les sols, par exemple), ainsi que les transferts d'usages des territoires (un site industriel converti en site d'habitation, par exemple). L'axe «Patrimoine» vise à étudier les impacts des polluants sur différentes cibles, à faire le point sur les modes de gestion des sols avec maintien ou changement d'usage et à comprendre les mécanismes de concertation sociale. Afin de croiser les connaissances acquises et de pouvoir communiquer plus facilement, un report cartographique des résultats est adopté afin de mettre en discussion les données.

\section{Contexte: un site multi-usages}

Le choix du site porte sur une mine dont le réaménagement a permis de développer plusieurs usages et qui présente la caractéristique d'avoir été un site d'extraction et de traitement de l'uranium. Ce site, dit de «La Commanderie », est le deuxième gisement uranifère de la Division de Vendée, avec 3978 tonnes d'uranium produites entre 1955 et 1991. La mine, qui dépendait de la concession de Mallièvre, est à cheval sur deux

\footnotetext{
${ }^{4}$ https://zatu.org/.
} 


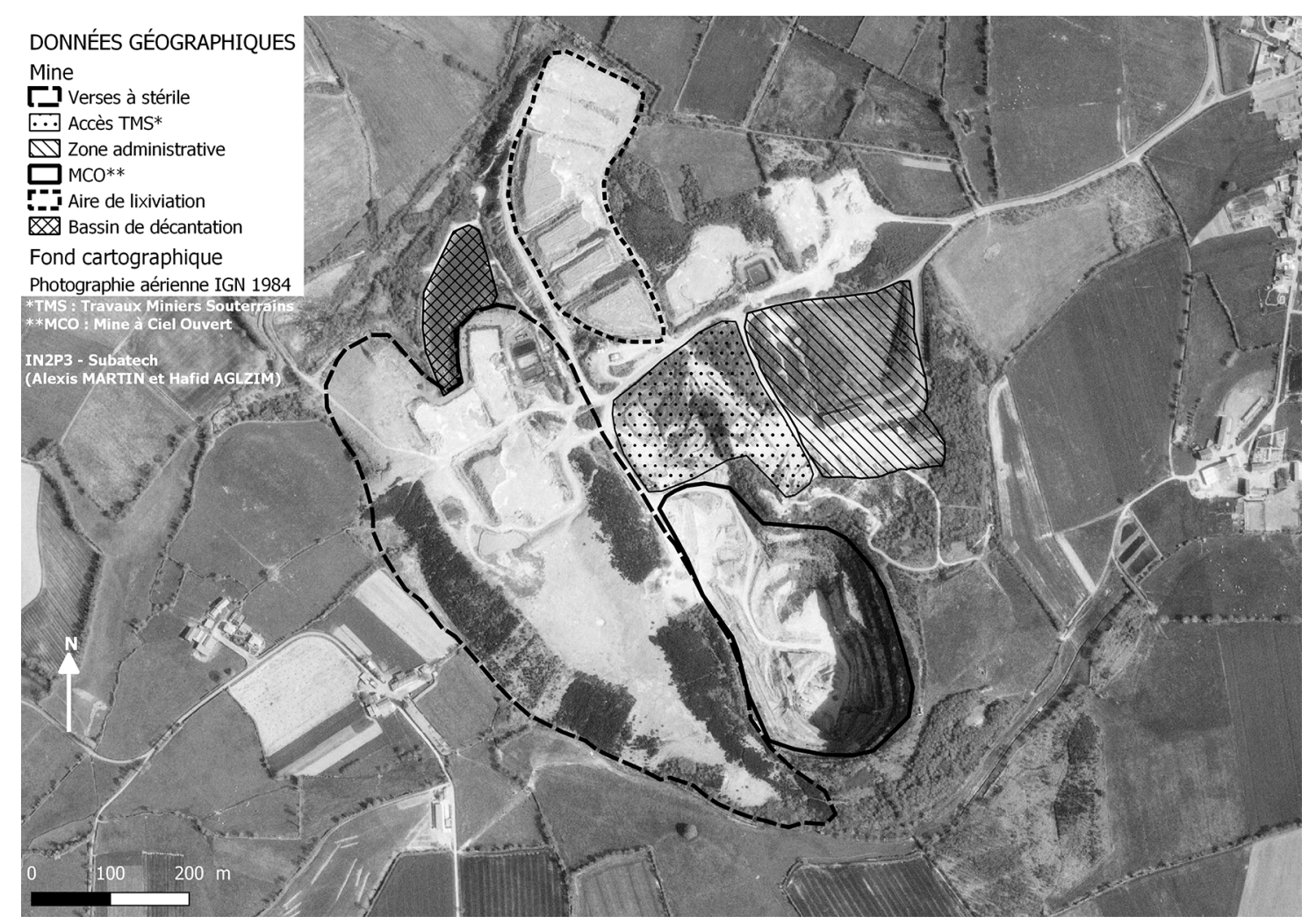

Fig. 1. Usage du foncier pendant l'exploitation (1984).

communes, Treize-Vents et Le Temple (associée à Mauléon depuis 1973), deux départements, la Vendée et les Deux-Sèvres, et deux régions, les Pays de la Loire et la Nouvelle-Aquitaine. Découverte en mai 1955 sur un terrain agricole, La Commanderie tire son nom d'un domaine éponyme fondé au début du XII ${ }^{\mathrm{e}}$ siècle, puis occupé par l'ordre monastico-militaire des Templiers. Elle représente le deuxième gisement de la Division de Vendée par sa production.

\section{Traces}

Le premier temps de la méthode a consisté en une identification physique des traces laissées par l'exploitation et en une mobilisation de l'histoire pour comprendre le marquage de certaines zones (Fig. 1). Un premier travail conduit autour des archives a permis d'identifier les zonages de l'exploitation en distinguant les zones de traitement du minerai, le stockage des stériles et la place des bâtiments. Dès 1955, les sondages préliminaires préparent la mise en exploitation intensive, avec une mine à ciel ouvert de $500 \mathrm{~m}$ de long, $250 \mathrm{~m}$ de large et $110 \mathrm{~m}$ de profondeur sur les deux départements, et des travaux souterrains longs de $30 \mathrm{~km}$ au total. Une verse à stériles de $200 \mathrm{~m}$ de large s'élève sur le côté ouest au fur et à mesure de l'extraction et des teneurs de coupure, tandis qu'au nord, les aires de lixiviation statique sont créées dès 1969. Les résidus de traitement, à base d'eau et d'acide sulfurique, sont déposés en tas au nord du site. L'exploitation à ciel ouvert s'arrête en 1977 tandis que les travaux souterrains prennent fin en 1983. Malgré les indices de gisements de pechblende vers le nord, la mine ferme en 1991. Elle a fourni au total 3978 tonnes d'uranium et produit 5,6 millions de tonnes de stériles. Il ne faut pas oublier que 250000 tonnes de résidus de lixiviation statique sont encore stockées sur site. L'analyse des archives a permis de spatialiser le passé industriel, notamment en identifiant les zones dédiées au traitement des résidus. Cette connaissance historique est d'un grand intérêt en radiochimie car elle permet de repérer les zones à risque. Par ailleurs, les éléments traces se trouvent réinscrits dans une histoire sociale et industrielle.

\section{Transfert}

Le deuxième temps de la méthode consiste à combiner les zones précédemment identifiées grâce à l'histoire, avec les données physico-chimiques. Les traces les plus simples à caractériser sont celles qui émettent les rayonnements lumineux (rayonnements gamma) et qui résultent des phénomènes de décroissance radioactive. Une sonde permettant de détecter ces rayonnements avec un système GPS intégré peut ainsi être utilisée. Une calibration au préalable permet de transformer la quantité de rayonnement détectée en 


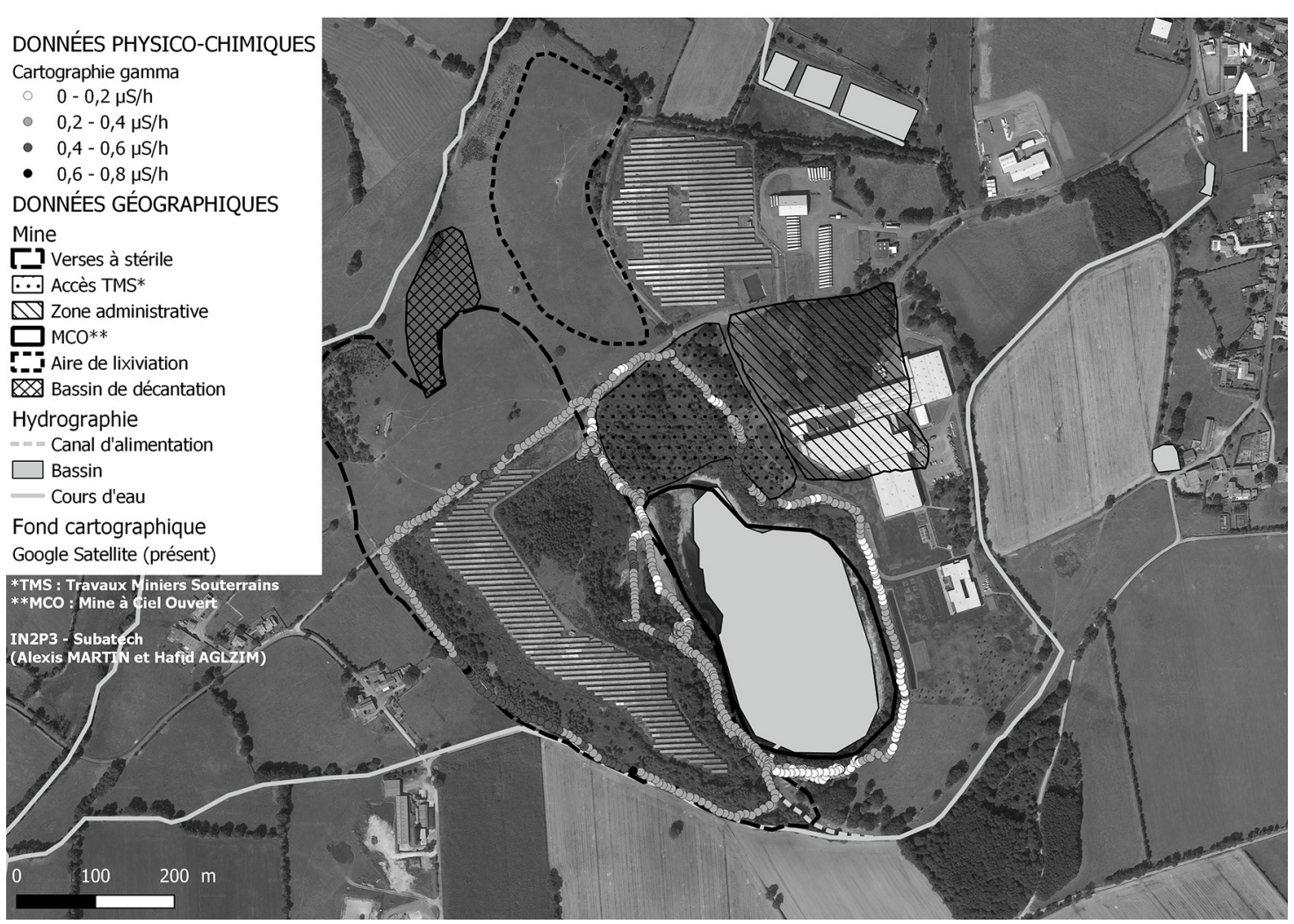

Fig. 2. Mesures et usages passés. Les résultats détaillés sont disponibles dans Martin et al. (2019).

dangerosité. Les traces que l'on peut rencontrer sont plutôt associées à des contaminations diffuses issues des termes sources et/ou à des contaminations issues de la période d'exploitation. Mais il est important de rappeler que l'on se trouve dans des environnements renforcés en radioactivité, et que des phénomènes complètement naturels peuvent conduire également à des processus de concentration et donc à des « anomalies » si l'on compare au fond géochimique ${ }^{5}$, dont on distingue la part «naturelle» (transformation de la roche et apports naturels) et la part «anthropique» (présence d'éléments due aux activités humaines).

La prise en compte de l'histoire est donc primordiale pour décorréler l'origine naturelle de l'origine industrielle. L'évaluation des risques implique en effet de prendre en compte les sources de contamination mais également l'identification des voies de transfert actives. Cette analyse requiert de comprendre les usages du site depuis l'exploitation. En effet, des changements d'usage peuvent avoir des impacts sur les équilibres établis au moment du réaménagement.

La figure 2 met précisément en relation les données physico-chimiques et les usages passés afin de caracté-

\footnotetext{
${ }^{5}$ Le fond géochimique est la composition chimique d'un sol et des roches sous-jacentes. Il est notamment utilisé dans les études des pollutions du sol.
}

riser les zones à risque. La superposition des deux éléments permet d'appréhender les mesures en faisant référence à des usages passés et d'aborder la question du transfert d'usage.

\section{Patrimoine}

Le troisième temps de la méthode vise à caractériser les usages inhérents au territoire en prenant en compte les pratiques, comme les zones de pâture ou d'irrigation.

Sur le site de La Commanderie, à partir de 1990, la fermeture de la mine à ciel ouvert a suscité de nombreuses sollicitations de reconversion auprès de l'ancien exploitant, notamment un projet de carrière qui n'a pas abouti. Finalement, pendant une vingtaine d'années, trois usages du territoire se combinent: le bassin d'eau de l'ancienne mine fait l'œuvre d'une demande du groupement agricole local pour être utilisé pour l'irrigation. Une société d'équipement et de conditionnement rachète les anciens locaux industriels et reconstruit un bâtiment neuf pour développer son activité. Enfin, sur l'ancienne verse à stériles, une société installe une centrale de panneaux photovoltaïques. Ces trois usages, énergétiques, industriels et agricoles cohabitent sur le site dont les élus successifs soulignent l'exemplarité en matière de reconversion. Parallèlement, au cours du réaménagement, trois périmètres de sécurité 


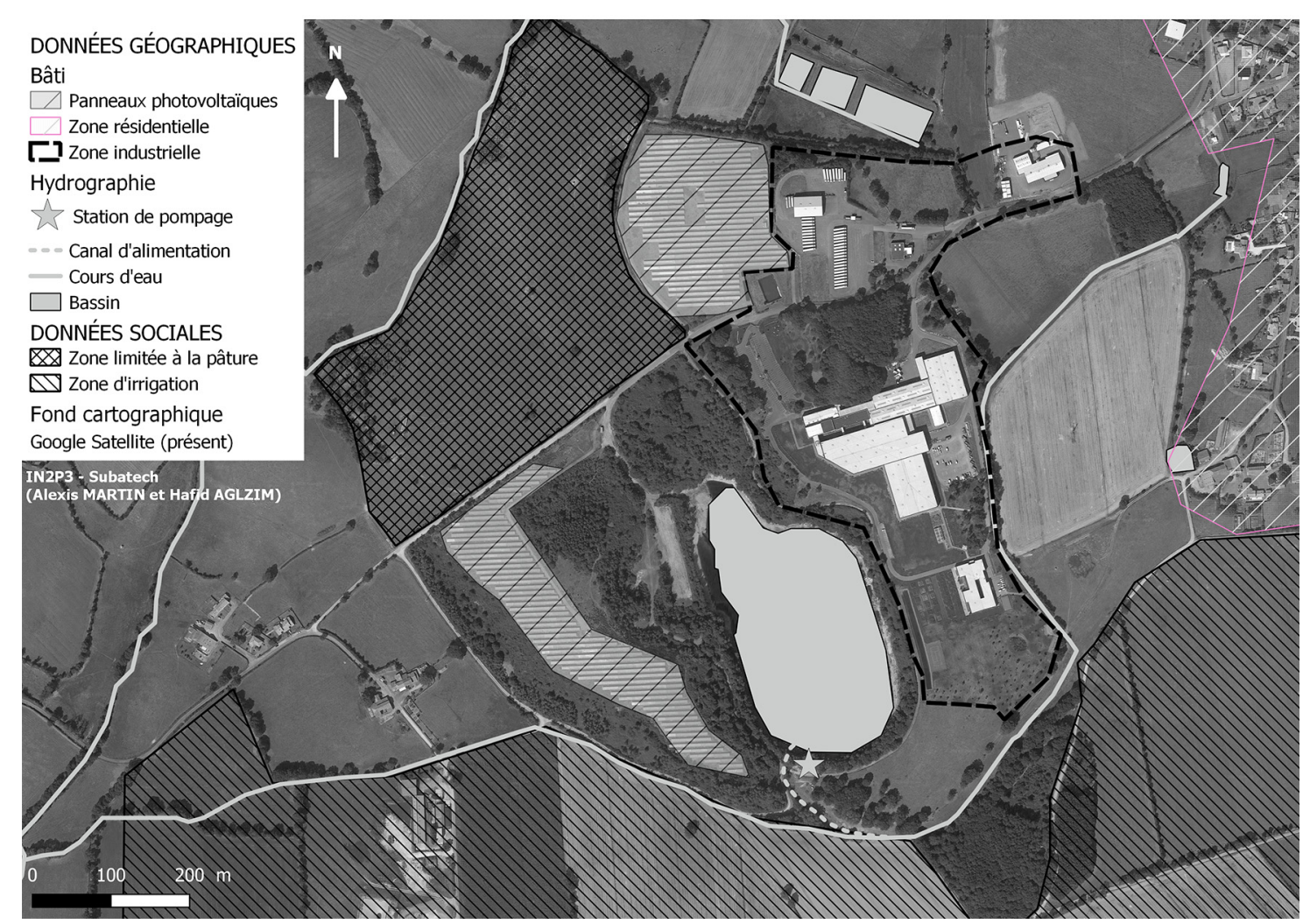

Fig. 3. Usages contemporains.

sont définis. La partie de la mine à ciel ouvert est remblayée avec des produits issus de l'assainissement et les résidus de lixiviation. L'ensemble des produits sont recouverts par un lit de calcaire d'une dizaine de centimètres d'épaisseur. La partie nord est comblée par des stériles miniers. La prise en compte des pratiques est majeure car sur le site, une controverse se développe au début des années 2000 autour de l'usage du bassin d'eau. En effet, un groupement de 16 exploitants agricoles l'utilise, alors que sur d'autres sites miniers, ces zones contenant des résidus sont considérées comme des installations classées pour la protection de l'environnement (ICPE). Au sein de la commission interdépartementale présidée par le préfet, qui se met en place à partir de 2009 pour gérer le site, les éléments liés aux impacts environnementaux sont cités ainsi que les éléments juridiques qui encadrent le stockage des résidus. Une gestion environnementale alignée sur la politique nationale est demandée par l'association locale de défense de l'environnement, qui de fait souhaite défendre une mise en œuvre cohérente avec l'ensemble des textes juridiques. Ce point est mis en discussion au sein de la commission interdépartementale, qui met en avant la nécessité de s'appuyer sur des analyses in situ. Une controverse s'installe entre l'association et les représentants des services de l'État qui s'appuient sur les usages du site et sa reconversion pour maintenir l'existant.
La figure 3 permet ainsi de caractériser les fonctions dédiées aux zones, leur compatibilité avec les relevés opérés et les usages locaux.

\section{Au-delà de la méthode : transmettre l'interdisciplinarité}

Le projet «Traces, Transfert, Patrimoine» se décline également sous la forme d'un enseignement délivré à l'IMT Atlantique et intitulé «Carturanium». Les élèves répondent à une demande, celle d'un maire qui souhaite évaluer les risques d'un site de la commune sur lequel résident des traces d'une ancienne mine d'uranium. Ils opèrent à la fois des prélèvements avec l'appui des radiochimistes, ils conduisent des enquêtes auprès des riverains et d'anciens mineurs et ils reportent à l'aide d'un système d'information géographique le produit des analyses. Leurs résultats sont ensuite présentés au maire de la commune et discutés en séance avec un jury composé d'enseignants-chercheurs et de «profanes ». Ils doivent ainsi évaluer le risque inhérent à ces traces d'uranium et proposer des modalités de gestion des territoires, en tenant compte des acteurs et de l'histoire locale.

Le corollaire du projet TTP porte également sur le développement d'une «science citoyenne», en capacité 
de répondre conjointement à des problématiques scientifiques, technologiques et sociétales. Il s'agit d'aborder les enjeux environnementaux à partir d'une démarche interdisciplinaire en lien avec les acteurs du monde industriel et associatif. En effet, un ensemble de dispositifs publics esquissent de nouvelles modalités de participation, voire de concertation. Conformément à l'esprit de la convention d'Aarhus (1998), les travaux initiaux du Groupe d'expertise pluraliste (GEP) sur les anciens sites miniers d'uranium du Limousin, menés de 2006 à 2010, et la circulaire ministérielle du 22 juillet 2009 sur la gestion des anciennes mines d'uranium insistent sur «l'accès à l'information et la participation du public aux processus décisionnels ». Toutefois, si ces travaux et ces textes participent à poser quelques principes pour un environnement concerté, ils ne fournissent aucun outil ni aucune méthode afin, dans un premier temps, d'obtenir un consensus sur l'évaluation des enjeux relatifs aux anciens sites miniers uranifères, et pour, dans un second temps, permettre de construire des dispositifs de réelle gestion concertée de ces enjeux et de ces sites sur le long terme. L'inscription des recherches scientifiques au cœur des enjeux locaux constitue une façon de garder un lien constant entre ces travaux et les acteurs qui y contribuent. Ainsi, dans ce cadre, les chercheurs engagés dans le projet TTP sont associés à l'une des commissions locales de suivi afin de donner à voir sur la scène citoyenne les résultats de recherche.

\section{La science du risque à la croisée des savoirs}

Si la singularité de l'héritage lié à l'exploitation de l'uranium porte sur son caractère diffus, invisible et très étendu dans le temps, le rapport à cet élément radioactif s'inscrit ainsi dans des systèmes locaux au sein desquels les représentations sociales vont fortement influencer la qualification des restes. En effet, la contamination se situe dans des territoires dans lesquels la structure de la propriété foncière, l'usage du site et le rapport à la souillure conditionnent le rapport local au risque. Par conséquent, appréhender le risque requiert d'étudier la dynamique de transfert des contaminants mais également les interactions qui ont lieu entre un environnement et ses habitants. Le double regard porté sur la gestion des mines d'uranium pose les conditions d'une prise en charge environnementale car c'est à la fois la connaissance des modes d'exploitation et de la composition de la matière qui permet d'appréhender les enjeux liés à la gestion à long terme. Cette approche permet de mettre en discussion l'objectivation par la mesure et les faits issus de l'histoire industrielle. Tout d'abord entre chercheurs, l'histoire éclaire et dimensionne la mesure physique, tout comme la mesure permet de réinterroger les usages sociaux de l'espace. Ces deux formes d'objectivation, certes, ne se combinent pas en termes de représentation mais elles permettent d'engager un dialogue itératif. D'autre part, la méthode rend compte de résultats ancrés dans la dynamique de territoire, ce qui crée les conditions d'une science ouverte aux questionnements des citoyens qui vivent sur ces sites.

Par conséquent, alors même que les débats scientifiques centrent leur propos sur la thématique très globale du développement durable, les enjeux associés aux risques environnementaux rappellent la nécessité d'une approche interdisciplinaire. En effet, le recours à la notion de risque environnemental conduit à couvrir une variété de problèmes caractérisés par la rencontre entre la sphère environnementale et la sphère des activités humaines. Au cœur de cette rencontre, se joue à la fois les questions de la mesure physique, du terme source et des impacts et celles de la perception du danger, de l'histoire d'un territoire ou de l'usage d'un site. De fait, les enjeux environnementaux invitent à renouveler les analyses par disciplines car ils se caractérisent par leur caractère systémique, la présence d'incertitudes et la prégnance du long terme. Les recherches sur l'environnement ont conduit les disciplines à aborder les objets qui leur étaient spécifiques, sous un angle nouveau, enrichi par les connaissances, méthodes et savoir-faire des autres disciplines.

Ce processus requiert de s'appuyer sur des pratiques fondées sur l'art des interfaces entre corpus disciplinaires et de fait, plusieurs formes de caractérisations du risque peuvent entrer en conflit. Par exemple, les modèles intégrateurs fondés sur la modélisation nécessitent de la part des disciplines de réduire, voire simplifier le spectre d'analyse. La seconde contrainte repose sur les échantillonnages et les échelles de temps. Les phénomènes d'accumulation, de transfert, les effets cocktails ou l'hybridation des contaminants seront potentiellement différents en fonction de l'objet d'étude et de son périmètre. Enfin, se joue dans la question de l'évaluation des risques, la hiérarchisation tacite des savoirs. Le terme d' "acceptabilité » souvent mobilisé dans les appels à projets pose d'emblée la variable sociétale comme une notion ad hoc distincte des autres savoirs. Par ailleurs, dans le cas de risques lents dont les effets s'échelonnent sur la longue durée, les incertitudes liées au passé rencontrent une pluralité d'évaluations, profanes ou expertes. La combinaison de regards entre disciplines peut s'avérer d'autant plus féconde lorsqu'elle contribue à la compréhension d'un phénomène multidimensionnel.

\section{Conclusion}

Le projet interdisciplinaire TTP illustre la volonté d'appréhender la notion de risque dans sa complexité en s'intéressant à un risque qui émerge en raison de 
changements d'usage du territoire. La question des risques dits "émergents » soulève des controverses au sein des communautés scientifiques, du fait de l'apparition de phénomènes nouveaux, parfois inédits, face auxquels les experts émettent des hypothèses et avis créant ou renforçant des clivages épistémologiques. La question des risques se pose aussi bien sur le volet de la complexité des facteurs d'émergence (nouveaux produits, nouvelles technologies) que sur la projection d'évènements non prévisibles dont les effets ne sont pas mesurables. Si l'interrogation sur l'interdisciplinarité porte souvent sur «la combinaison des savoirs» pour répondre à un enjeu commun, les pratiques sont souvent le parent pauvre du questionnement. Néanmoins, la thématique du risque environnemental invite chaque chercheur à s'inscrire dans une dynamique de dialogue et de traduction de ses résultats vis-à-vis de ses collègues des autres disciplines.

\section{Références}

Barthe Y., 2006. Le pouvoir d'indécision. La mise en politique des déchets nucléaires, Paris, Economica.

Becerra S., Lalanne M., Weisbein J. (Eds), 2016. Faire face aux risques dans les sociétés contemporaines, Toulouse, Octarès Éditions.
Bretesché S., Ponnet M., 2012. Le risque au défi de la mémoire organisée. L'exemple de la gestion des mines d'uranium françaises. Vertigo. La revue électronique en sciences de l'environnement, 12, 1, http://journals.openedition.org/ vertigo/11992, https://doi.org/10.4000/vertigo.11992.

Bretesché S., Ponnet M., 2013. Le risque environnemental entre oubli et gestion du passé. Le cas d'une ancienne mine d'uranium française. Annales des Mines - Gérer et comprendre, 111, 1, 15-24, https://www.cairn.info/revuegerer-et-comprendre1-2013-1-page-15.htm, https://doi.org/ 10.3917/geco.111.0015.

Brunet P., 2004. La nature dans tous ses états. Uranium, nucléaire et radioactivité en Limousin, Limoges, Presses universitaires de Limoges.

Dauphiné A., Provitolo D., 2013. Risques et catastrophes, Paris, Armand Colin.

Hecht G., 2016. Being nuclear. Africans and the global uranium trade, Cambridge (MA), MIT Press.

Martin A., Landesman C., Lépinay A., Roux C., Champion J., Chardon P., Montavon G., 2019. Flow period influence on uranium and trace elements release in water from the waste rock pile of the former La Commanderie uranium mine (France), Journal of Environmental Radioactivity, 208-209, 106010, https://doi.org/10.1016/j.jen vrad.2019.106010.

Thom R., 1993. Prédire n'est pas expliquer, Paris, Flammarion.

Citation de l'article : Bretesché S., Montavon G., Martin A. Pour une approche interdisciplinaire du risque environnemental. Le cas de l'uranium. Nat. Sci. Soc. 28, 1, 58-65. 